\title{
Distant Plasma Sheet Ion Distributions during Reconnection
}

\author{
C. J. Owen, R. T. Mist \\ Mullard Space Science Laboratory, University College London, UK
}

\begin{abstract}
Previous models of the plasma sheet following reconnection and current sheet acceleration predict 'lima-bean' ion distributions. These are inconsistent with observational constraints. We postulate that following initial interaction with the current sheet, a fraction of outflow ions are backscattered and re-encounter the current sheet. Fermi acceleration processes then generate an additional high-energy outflow population. In the backscatter region these ions form a complete shell in velocity space, providing sufficient pressure to support weak fields. Further from the current sheet, where backscatter is less efficient, a hemispherical shell of ions moves away from the current sheet, and field strengths are nearer the external lobe value. The fastest ions stream ahead of the bulk population to form the plasma sheet boundary layer. This model predicts a multi-layered plasma sheet structure, consistent with recent GEOTAIL observations.
\end{abstract}

\section{Introduction}

In the distant tail, a 1-d approximation can be used to study current sheet acceleration following reconnection. Here, field forces are balanced by pressure anisotropy [Rich et al., 1972]. Early studies [see Cowley, 1984] show the field-aligned outflow beam has speed of order the external lobe Alfvén speed. This plasma occupies the central portion of the reconnected field wedge downstream from the neutral line, and does not extend to the magnetic separatrix [Hill, 1975]. However, higher energy particles may stream ahead of the beam to form a separatrix layer, the deep-tail plasma sheet boundary layer (PSBL), beyond the beam edge [e.g. Cowley, 1984]. Observations compatible with these models have been reported [e.g. Cowley et al., 1984]. However, close correlations between observed plasma sheet flow speeds and the expectations of the models are not found [Mist and Owen, 1999]. Moreover, [Hoshino et al., 2000] observe a multi-layered plasma sheet structure and describe the plasma sheet as a nested set of MHD slow mode shocks and contact discontinuities, which create an inner and outer plasma sheet. Their observations show that particle distributions can exhibit significant structure throughout the plasma sheet.

\section{Copyright 2001 by the American Geophysical Union.}

Paper number 2001GL013032.

0094-8276/01/2001GL013032\$05.00
In this paper, we address the structure of the plasma sheet distributions. We illustrate the shortcomings of previous current sheet acceleration models for the distant plasma sheet. We postulate a region adjacent to the current sheet, where wave-particle interactions or turbulence result in significant backscatter of the outflow beam. We show the resulting high-energy ions play a vital role in overall pressure balance. We also recover a multi-layered plasma sheet consistent with Hoshino et al. [2000], and a natural explanation for the origin of the high-energy particles in the deep tail PSBL.

\section{Previous Models}

The general stress balance equations for a 1-d current sheet are [e.g., Owen and Cowley, 1987]

$$
P_{\perp}+\frac{B^{2}}{2 \mu_{0}}=K ; \quad M_{\|}-P_{\perp}=\frac{B^{2}}{\mu_{0}}
$$

$M_{\|}$and $P_{\perp}$ are the plasma stress tensor components parallel and perpendicular to the field, $B$ is the field strength outside the current sheet and $K$ is a constant. Both parallel pressure and momentum associated with bulk field-aligned flow are included in $M_{\|}$. For a cold incoming lobe plasma population undergoing acceleration and heating at the current sheet, these become

$$
\begin{aligned}
P_{\perp P S} & =\frac{B_{L}^{2}-B_{P S}^{2}}{2 \mu_{0}} \\
M_{\|_{T O T}} & =M_{\|_{L}}+M_{\|_{P S}}=\frac{B_{L}^{2}+B_{P S}^{2}}{2 \mu_{0}} .
\end{aligned}
$$

Here $B_{L}$ and $B_{P S}$ are the lobe and plasma sheet field strengths, $M_{\|_{L}}$ is the parallel momentum flux of the lobe inflow and $P_{\perp P S}$ and $M_{\|_{P S}}$ are the perpendicular pressure and parallel momentum flux of the plasma sheet outflow. Although these can be used to predict the macroscopic plasma properties in terms of $B_{L}$ and $B_{P S}$, they do not address particle level processes.

Cowley and Shull [1983] (hereafter C-S) proposed a simple model of ion distributions following current sheet acceleration. The principles of this model are illustrated in Figure 1, panels (a) and (b). These indicate the positions of the inflow and outflow populations in the field line rest frame (FLRF). Each panel shows velocity space, with the unprimed axes $\left(V_{x}, V_{z}\right)$ representing the Earth rest frame (ERF) and the primed axes $\left(V_{x}{ }^{\prime}, V_{z}^{\prime}\right)$ the FLRF. The two frames are separated by $V_{F}$, the reconnected field line contraction speed in the ERF. The 

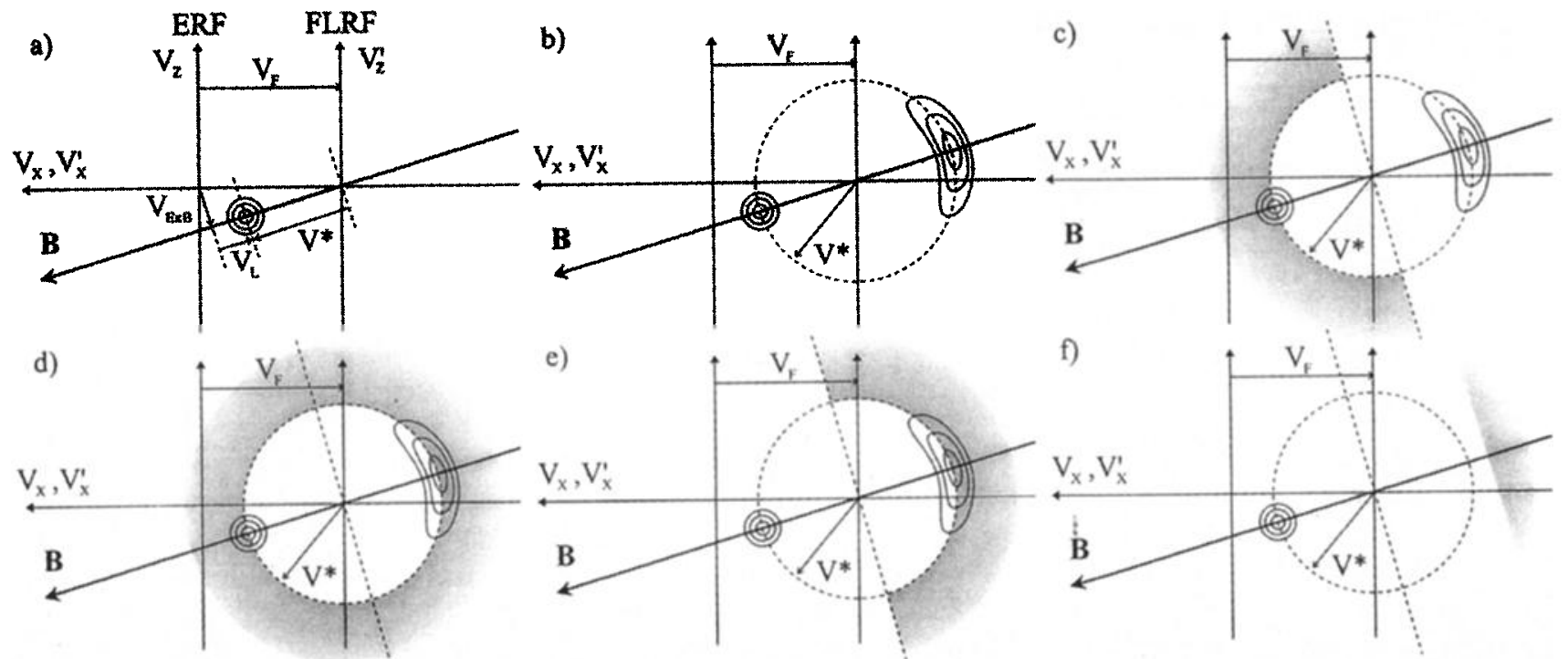

Figure 1. Velocity space diagrams illustrating the construction of deep tail plasma sheet distributions. Cold plasma inflow from the lobes (a) is first turned around and scattered to form a Cowley-Shull type distribution (b). Backscatter in the inner plasma sheet creates a higher energy population heading back to the current sheet (shaded in (c)). After further interaction with the current sheet, a full spherical shell of energetic ions is created (d). In the outer plasma sheet, where scattering is unimportant, only outflowing energetic ions are observed with the Cowley-Shull and inflow populations (e). At the outer extremes of the reconnected field lines, only the fastest outflow ions are observed (f). Distributions (a), (f), (e) and (d) represent the plasma populations in the lobe, plasma sheet boundary layer, outer and inner plasma sheet regions respectively.

$V_{x}, V_{x}^{\prime}$ direction corresponds to motions parallel to the current sheet and the $V_{z}, V_{z}^{\prime}$ directions motion normal to this surface. The B-field just northward of the current sheet is indicated by the heavy arrowed lines. During reconnection, cold plasma $\mathbf{E} \times \mathbf{B}$ drifts from the surrounding lobes toward a thin current sheet (panel (a)). In the ERF, the bulk motion of this distribution consists of velocities $V_{E \times B}$ perpendicular to $\mathbf{B}$, and $V_{L}$ antiparallel to $\mathbf{B}$ in this example. This distribution has a parallel velocity $V^{*}$ in the FLRF. In this frame, the electric field is transformed away [de Hoffman and Teller, 1950] and the ion kinetic energy is conserved. Thus after reflection at the current sheet, ions are scattered around the dotted circle of radius $V^{*}$ (panel (b)). In the ERF, this outflow emerges as a broadened beam of ions, with bulk field-aligned speed $\sim 2 V^{*}$ greater than the lobe population. C-S treat a simplified case of a distribution spread evenly up to a limiting pitch angle, $\alpha_{B}$, and obtain plasma stress tensor components:

$$
\begin{aligned}
M_{\|_{L}} & =m_{i} n_{L} V^{*^{2}}=m_{i} n_{P S} V^{*^{2}} \cos ^{2} \frac{\alpha_{B}}{2} \\
M_{\|_{P S}} & =\frac{m_{i} n_{P S} V^{*^{2}}}{3}\left(1+\cos \alpha_{B}+\cos ^{2} \alpha_{B}\right) \\
P_{\perp P S} & =\frac{m_{i} n_{P S} V^{*^{2}}}{3}\left(1-\frac{\cos \alpha_{B}}{2}-\frac{\cos ^{2} \alpha_{B}}{2}\right)
\end{aligned}
$$

For a given $V^{*}, P_{\perp P S}$ is a maximum for the limiting case $\alpha_{B}=\pi / 2$, corresponding to an outflow distribution spread uniformly around a hemisphere of radius $V^{*}$ in the region $V_{\|}<0$. This corresponds to a minimum of $M_{\|_{T O T}}$, and so $M_{\|_{T O T}} / P_{\perp P S} \geq 5 / 2$. Using (3) and (4), it follows that this mechanism can balance the plasma sheet stresses if $B_{P S}>\sqrt{ }(3 / 7) B_{L}$. In reality, we expect partial scattering, with fewer ions at higher pitch angles, such that $\alpha_{B}<\pi / 2$ and $B_{P S} \gg \sqrt{ }(3 / 7) B_{L}$. Slavin et al. [1985] report $B_{L}=9.2 \mathrm{nT}$ on average, implying that $B_{P S} \gg 6.0 \mathrm{nT}$. However, Slavin et al. also report an average $B_{P S}=4.0 \mathrm{nT}$. Moreover, the B-field strength is highly variable, often approaching zero close to the current sheet. Typically, the C-S process provides insufficient $P_{\perp}$ to satisfy (3) for a value of $V^{*}$ determined to satisfy condition (4). Consequently, outflow beam ions must either be preferentially scattered to perpendicular pitch angles, or further modification of the heating process is required. In addition, this picture lacks a description of the origin of higher-energy particles which form the PSBL, nor does it account for spatial structure within the plasma sheet itself. We now adapt this model, in order to remove these discrepancies and provide an improved link to the observations.

\section{Proposed Velocity Space Structure}

Figure 1 illustrates the proposed origins of the different populations in the plasma sheet distribution. Each panel illustrates an aspect of the formation of the overall distribution, not a time evolution, so not all represent observable distributions. At the current sheet, the incident lobe population first undergoes the C-S process, and is turned around, accelerated and heated (panels a, b). We propose that in a region adjacent to the current sheet, backscattering of some of the accelerated outflow ions occurs. This may be due to field direction fluctuations in turbulent magnetic structures or wave-particle interactions. We assume the scattering may be represented by a random series of reflecting surfaces moving at speeds $\leq V_{F}$ in the ERF. Ions encountering one of 
these surfaces during outflow from the current sheet are re-reflected. They conserve kinetic energy in the rest frame of their scattering surface. Thus they gain energy in the FLRF and appear with speeds $>V^{*}$, moving back toward the current sheet $\left(V_{\|}>0\right)$. Assuming they also pitch angle scatter, they appear in the shaded region in panel (c). These ions now re-encounter the current sheet, where they again reflect and scatter about the FLRF origin. Consequently, just outside the current sheet, we expect a full shell of multiply reflected ions with FLRF speeds $>V^{*}$ as well as the unmodified lobe and singly-reflected C-S populations (panel (d)). In effect, a high-energy population appears as a fraction of the plasma sheet outflow experiences Fermi acceleration between the current sheet and scattering surfaces.

We assume the backscattering region is of limited extent, or that the backscatter probability falls off with distance from the current sheet. The flux of backscattered ions returning to the current sheet is high close to the current sheet, and vanishes at its outer edge. Thus the outer plasma sheet contains only the inflow, and those parts of the distribution heading away from the current sheet (antiparallel to B) in the FLRF (panel (e)). Velocity filter effects confine the C-S population to the central portion of the reconnected field wedge, defining the edge of the outer plasma sheet. Beyond this, extending towards the separatrices, only the lobe inflow and those multiply-reflected ions with the fastest field-aligned outflow speeds will be found (panel $(f)$ ). Hence this process naturally explains the origin of highenergy particles in this layer, which corresponds to the PSBL [Cowley et al., 1984].

In practice, some ions undergo many interactions with the current sheet. However, such ions remain in the shaded regions in Figure 1, so panels (c) - (e) implicitly include this possibility. We have also ignored any Fermi deceleration within the backscatter region. This would lead to some ions scattering inside the circle of radius $V^{*}$ in the FLRF.

To illustrate the effects of this process we consider a quantitative example where the observed average lobe field strength $(9.2 \mathrm{nT})$ reduces to the average plasma sheet field (4.0 nT) at the current sheet. We assume $n_{L}=0.3 \mathrm{~cm}^{-3}$, an initial scattering via the $\mathrm{C}-\mathrm{S}$ process to a limiting pitch angle $\alpha_{B}=60^{\circ}$, and that the subsequent scattering process reflects $10 \%$ of outflow particles crossing the inner plasma sheet. The field strength at the current sheet implies $P_{1 P S}=27.3 \mathrm{pPa}$ while $M_{\|}-P_{\perp}=12.7 \mathrm{pPa}$. At the current sheet, the multiplyreflected, high-energy populations are isotropic, so only the inflow and C-S distributions contribute to $M_{\|}-P_{\perp}$. For the assumed $n_{L}$ and $\alpha_{B}$ this condition is satisfied by $V^{*}=134 \mathrm{~km} \mathrm{~s}^{-1}$ ( the combined inflow and C-S distributions have $M_{\|}=19.2 \mathrm{pPa}$ and $P_{\perp}=6.5 \mathrm{pPa}$; thus the isotropic high-energy population has $P_{\perp}=20.8 \mathrm{pPa}$ ). This value is significantly below that from cold plasma models [e.g. Cowley, 1984] where $V^{*}=260 \mathrm{~km} \mathrm{~s}^{-1}$ (the external lobe Alfvén speed). The bulk field-aligned outflow speed in the ERF is $V_{F}=\left(V^{*}+V_{L}\right)$, where the typical external lobe flow speed, $V_{L} \sim 100 \mathrm{~km} \mathrm{~s}^{-1}$.

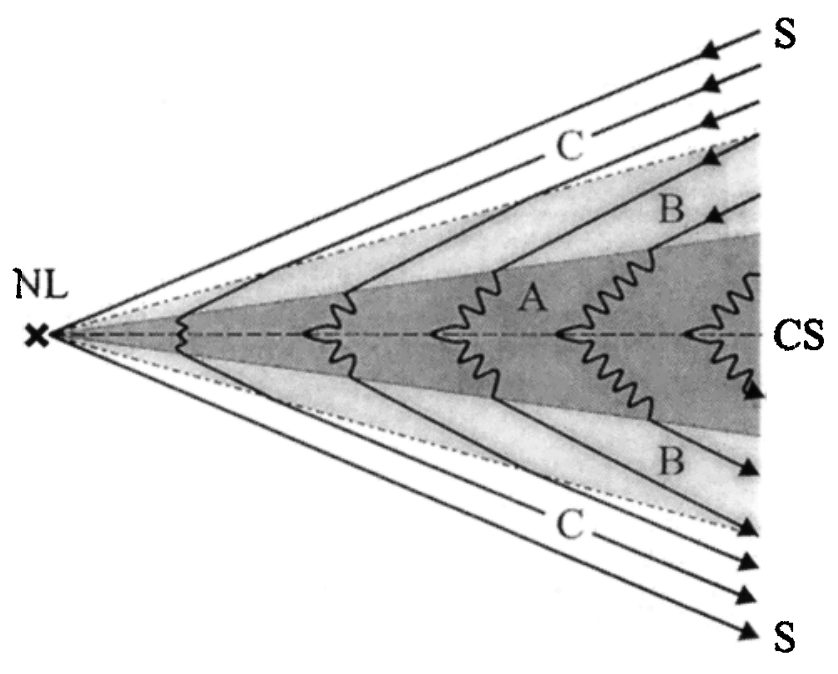

Figure 2. Plasma sheet spatial structure downstream of a reconnection neutral line (NL) based on this model. Adjacent to the current sheet (CS), the inner plasma sheet (A) contains backscattered ions returning to the current sheet, supporting weak and turbulent fields. The outer plasma sheet (B) is devoid of backscattered ions and is associated with higher field strengths. The fastest outflow jons extend toward the separatrix (S) creating the PSBL (C).

Thus this model predicts bulk plasma sheet flow speeds $\sim 234 \mathrm{~km} \mathrm{~s}^{-1}$, compared to $360 \mathrm{~km} \mathrm{~s}^{-1}$ in cold plasma models. This may provide an improved match to the observed flow speeds, which are overestimated by cold plasma models [Mist and Owen, 1999].

At the edge of the scattering region, we expect in this example an outflow comprising $90 \%$ of the C-S distribution and $90 \%$ of the outflow half of the high-energy distribution. These ions provide $P_{\perp} \sim 0.9(6.5+20.8 / 2)$ $=15.2 \mathrm{pPa}$. To maintain overall perpendicular pressure balance, $B_{P S} \sim 6.8 \mathrm{nT}$ at this point. In this example, the inner plasma sheet, or scattering region, is thus defined by field strengths $4<B_{P S}<6.8 \mathrm{nT}$. The outer plasma sheet occupies the region where $B_{P S}>6.8 \mathrm{nT}$, out to the furthest extent reachable by ions in the $\mathrm{C}-\mathrm{S}$ distribution under the velocity filter effect. $\mathrm{Be}$ yond this point, high-energy ions with $\left|V_{\|}\right|>V^{*}$ form the deep tail PSBL. We assume the high-energy ion distribution has a power-law dependence on ion energy, $f \propto E^{-\gamma}(\gamma>5 / 2)$. Taking $\gamma=3$, ions with $\left|V_{\|}\right|>V^{*}$ have $P_{\perp} \sim 3.5 \mathrm{pPa}$ just outside the outer plasma sheet, such that pressure balance requirements imply $\mathrm{B}=8.7 \mathrm{nT}$. Hence, the outer plasma sheet in this example is associated with field strengths $6.8<B_{P S}<$ $8.7 \mathrm{nT}$. Finally, the high-energy ions in the velocity dispersed PSBL/separatrix layer are associated with field strengths $8.7<B_{P S B L}<B_{L}(=9.2 \mathrm{nT})$.

\section{Summary and Discussion}

We have suggested an adaptation to previous models of current sheet acceleration following reconnection in the tail. This is based on secondary processing of some of the heated and accelerated plasma sheet outflow following backscatter in the inner plasma sheet. These 
ions undergo Fermi acceleration as they bounce between the scattering surface and the current sheet, thus creating a high-energy population within the plasma sheet.

The spatial structure of the fields and plasma populations resulting from the model is illustrated in Figure 2. The vertical scale is expanded with respect to the horizontal. Reconnection at a neutral line (NL) creates a hairpin-like field geometry. Field lines mapping instantaneously back to the NL define the magnetic separatrices (S). Outside of these, the field has not yet reconnected, so these regions contain only open magnetic flux and cold inflow plasma (c.f. Figure 1(a)). Between the separatrices, the main reversal of the reconnected field occurs at the central current sheet. This is the primary site of acceleration and heating of inflow plasma in this model. The resulting outflow plasma jets mainly along the average field direction away from the current sheet on both sides.

Adjacent to the current sheet, we expect an inner plasma sheet (Region A in Figure 2) in which a full shell of energetic ions co-exists in velocity space with the 'lima-bean' type outflow population previously suggested by Cowley and Shull [1983] (c.f. Figure 1(d)). The high-energy ions provide the pressure to support very low field strengths in this region. The field may also show fluctuations related to the scattering surfaces postulated above. Further from the current sheet, where backscatter is weaker, we expect an outer plasma sheet (Region B), containing only those ions that have escaped the scattering region. A hemispherical shell of high-energy ions moving away from the current sheet appears with the 'lima-bean' population (c.f. Figure 1(e)). These ions support only a moderate field strength reduction from that in the external lobes. The outer edge of the outer plasma sheet is the boundary of those ions having undergone a single encounter with the central current sheet. Beyond this, but inside the magnetic separatrices (Region C), only those multiply-reflected energetic ions with the greatest field-aligned outflow speeds will be observed with the lobe inflow (c.f. Figure $1(f)$ ). This region represents the velocity-dispersed PSBL, where the B-field is reduced only slightly from that of the adjacent lobes. Hence 3 main spatial regions naturally arise, an inner and outer plasma sheet and the PSBL. We note that the boundaries between these regions may be the layers observed by Hoshino et al. [2000], who identified them as an MHD slow-shock and contact discontinuity pair. Although sharp boundaries are drawn between regions in Figure 2, the transitions are likely to be more gradual as the plasma evolves with distance from the current sheet. Note also that, since the initial acceleration and heating occurs at the central current sheet in this model, we predict that the lobe inflow co-exists with the outflow populations within each plasma sheet region.

Note that we do not rule out either the possibility that the field strength at the central current sheet may fall to almost zero, or that the fraction of outflow ions reflected within the inner plasma sheet may approach unity. The former case results in $V^{*} \sim 0$, such that the void within the centre of the ring distribution and the distinction between the inflowing lobe and outflowing 'lima-bean' distribution may be indiscernible. In the latter case, the outflowing 'lima-bean' distribution may become indistinguishably weak in comparison to the enhanced fluxes within the energetic ring distribution. Either of these factors could account for reports of near-isotropic distributions near the central current sheet [Hoshino et al., 2000]. Also, one or more of the populations may lie below an instrument threshold, or outside an energy range. We do not therefore claim that all components should always be observed. Note also that the presented example is specific to the parameter values assumed, although we employed typical numbers. However, that analysis can be employed for any given plasma sheet observation, and details of the observed and predicted distributions directly compared to verify this model.

Acknowledgments. CJO and RTM were supported by a UK PPARC Advanced Fellowship and Post-Doctoral Research Assistantship respectively.

\section{References}

Cowley, S. W. H., The distant geomagnetic tail in theory and observation, Magnetic Reconnection in Space and Laboratory Plasmas, ed. E. W. Hones Jr., vol. 30, Geophys. Monogr. Series, 228, AGU, Washington, DC, 1984. Cowley, S. W. H., and P. Shull, Current sheet acceleration of ions in the geomagnetic tail and properties of ion bursts at the lunar distance, Planet. Space. Sci, 31, 235, 1983.

Cowley, S. W. H., R. J. Hynds, G. Richardson, P. W. Daly, K. P. Wenzel, J. A. Slavin, and B. T. Tsurutani, Energetic ion regimes in the deep geomagnetic tail - ISEE-3, Geophys. Res. Lett., 11, 275, 1984.

de Hoffman, F., and E. Teller, Magneto-hydrodynamic shocks, Phys. Rev., 80, 692, 1950.

Hill, T. W., Magnetic merging in a collisionless plasma, $J$. Geophys. Res., 80, 4869, 1975.

Hoshino, M., T. Mukai, I. Shinohara, and Y. Saito, Slow shock downstream structure in the magnetotail, $J$. Geophys. Res., 105, 337, 2000.

Mist, R. T., and C. J. Owen, ISEE-3 observations of slow flows in the magnetotail plasma sheet, J. Geophys. Res., $104,25,063,1999$.

Owen, C. J., and S. W. H. Cowley, A note on current sheet stress balance in the geomagnetic tail for asymmetrical lobe plasma conditions, Planet. Space. Sci, 35, 467, 1987.

Rich, F. J., V. M. Vasyliunas, and R. A. Wolf, On the balance of stresses in the plasma sheet, J. Geophys. Res., 77, 4670, 1972.

Slavin, J. A., E. J. Smith, D. G. Sibeck, D. N. Baker, R. D. Zwickl, and S. I. Akasofu, An ISEE 3 study of average and substorm conditions in the distant magnetotail, $J$. Geophys. Res., 90, 10,875, 1985.

C. J. Owen and R. T. Mist, Mullard Space Science Laboratory, Holmbury St. Mary, Surrey, RH5 6NT, England. (email: cjo@mssl.ucl.ac.uk; rtm@mssl.ucl.ac.uk)

(Received February 15, 2001; revised April 26, 2001; accepted May 7, 2001.) 